\title{
The observations and analyses of an eruptive prominence
}

\author{
L. Kejun ${ }^{1,2}$ and Z. Shuhua ${ }^{2}$ \\ ${ }^{1}$ CCAST(World Laboratory), Beijing P.O. Box 8730, Beijing 100080, P.R. China \\ 2 Yunnan Observatory, the Chinese Academy of Sciences, P.R. China*
}

Received July 11; accepted November 7, 1996

\begin{abstract}
The tornado-shaped eruptive prominence on 29 August 1990 has been analysed in three aspects in this paper: morphological evolution, spectral diagnosis and kinematic characteristics. It is found that: (1) the altitude of the prominence uniformly increased, then evenly decreased. The rising velocity of the altitude of the prominence was about twice as large as the falling velocity. (2) the materials in the prominence spiraled to rise, then after reaching the climax, they dropped in succession. The observations showed that the flux ropes were already twisted when they emerged. (3) During the period of the development of the prominence, three loop structures were formed, with intermittent material ejection. The shape of an eruptive prominence and its changes with time could be an important factor for studying properties of eruptive prominences. The observations have also been explained qualitatively in this paper.
\end{abstract}

Key words: Sun: activity — Sun: prominence, flare

\section{Introduction}

Solar filaments are long thin threads of relatively dense cool mostly-ionised gas suspended in the million-degree totally-ionised corona. Because filaments are about a hundred times denser than the ambient corona, they should submerge into the chromosphere below, where the density and temperature are similar to their own. Instead, filaments can remain suspended in the corona for a long time, and it is generally accepted that peculiar magnetic fields support them against the pull of gravity and insulate them from the heat of the corona (Rust \& Kumar 1994; Hirayama 1979, 1985; Poland 1986; Priest 1989; Ruzdjak \& Tandberg-Hanssen 1990).

Prominences are filaments seen at the solar limb, but they are seen in two-dimensions, and can be more usefully studied. Whether prominences or filaments, they

\footnotetext{
mail address: Yunnan Observatory, Kunming P.O. Box 110,
} P.R. China. have many unknowns: source of the material, formation etc., and further investigation is necessary. On 29 August 1990, we observed an eruptive prominence, and a series of $\mathrm{H}_{\alpha}$ filtergrams and $\mathrm{H}_{\gamma}$ spectra were obtained. In this paper, the prominence will be studied to reveal its structure, evolution and kinematic characteristics in detail.

\section{The observations and analyses of the morphological evolution}

\subsection{Observations}

On 29 August 1990, a $1 \mathrm{~N}$ flare occurred in the region

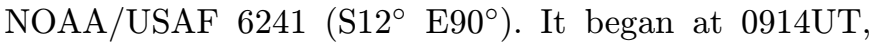
reached a maximum at 0920UT and ended at 0940UT (NOAA 1990a). Companying the flare, a tornado-shaped eruptive prominence (S12; E90) occurred over the flare. The prominence began at 0917UT and ended at 0959UT (NOAA 1990b). The prominence began after the begining of the flare, and ended later than the flare. Although a type III radio burst was recorded for the event, we can hardly say anything more about the relation of the prominence with the burst, because of the prominence accompanying the flare and imperfections of the obtained data. No X-ray radiation was detected with GOES.

A series of $\mathrm{H}_{\alpha}$ filtergrams of the prominence in the interval 0919 - 0947UT was obtained at Yunnan Observatory by using $\mathrm{H}_{\alpha}$-SSHG (Xuan \& Lin 1993). Twodimensional $\mathrm{H}_{\gamma}$ spectral data in the period 0927-0931UT were obtained as well. We obtained a total of $42 \mathrm{H}_{\gamma}$ images. These two kinds of data are shown in Fig. 1.

\subsection{The morphological evolution}

Figure 1 shows a regular loop structure before 0919UT. The axis of the loop and the solar surface are declined about $60^{\circ}$ toward the north. The first and second filtergrams in the figure indicate the emerging of a loop structure, i.e. a magnetic flux tube, and seem to show a twist of the left loop leg; the flux rope emerged already twisted. The later spectral diagnoses will confirm this result. The same result was obtained by Tanaka (1991) 

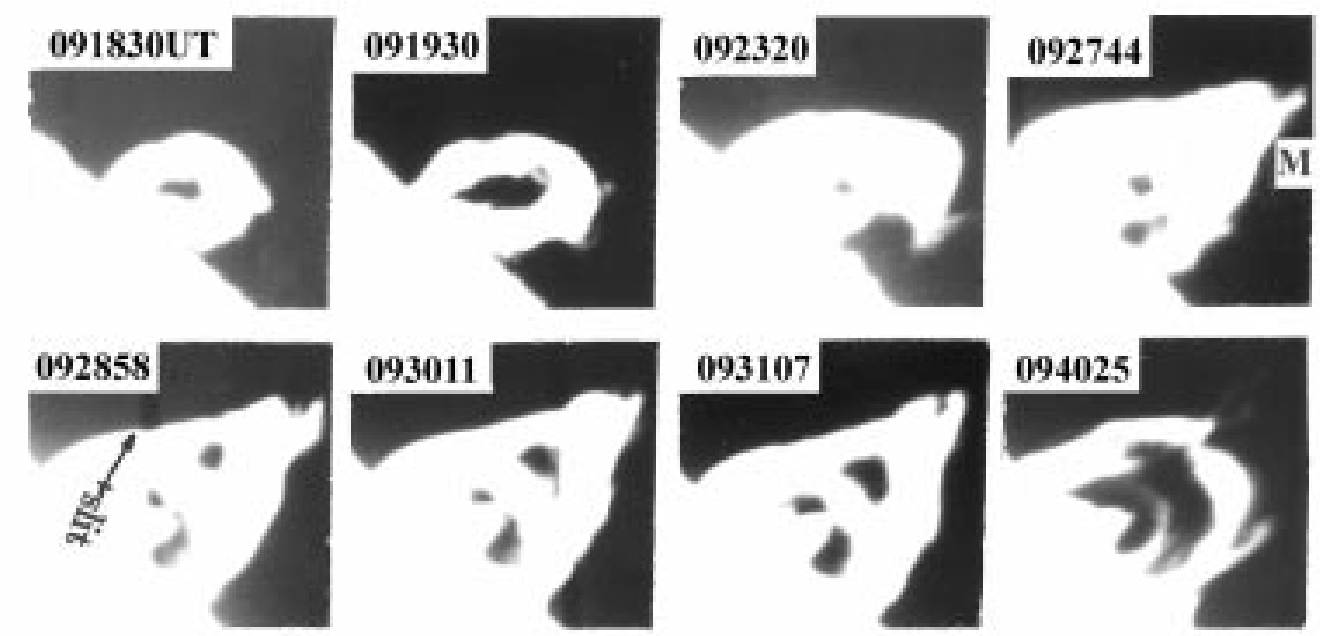

\section{5}
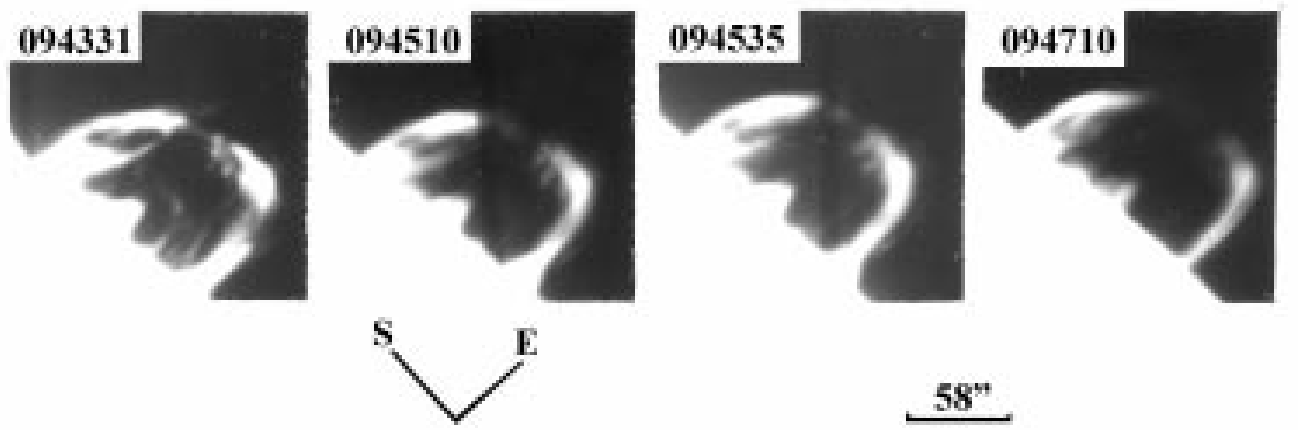

$58^{n}$
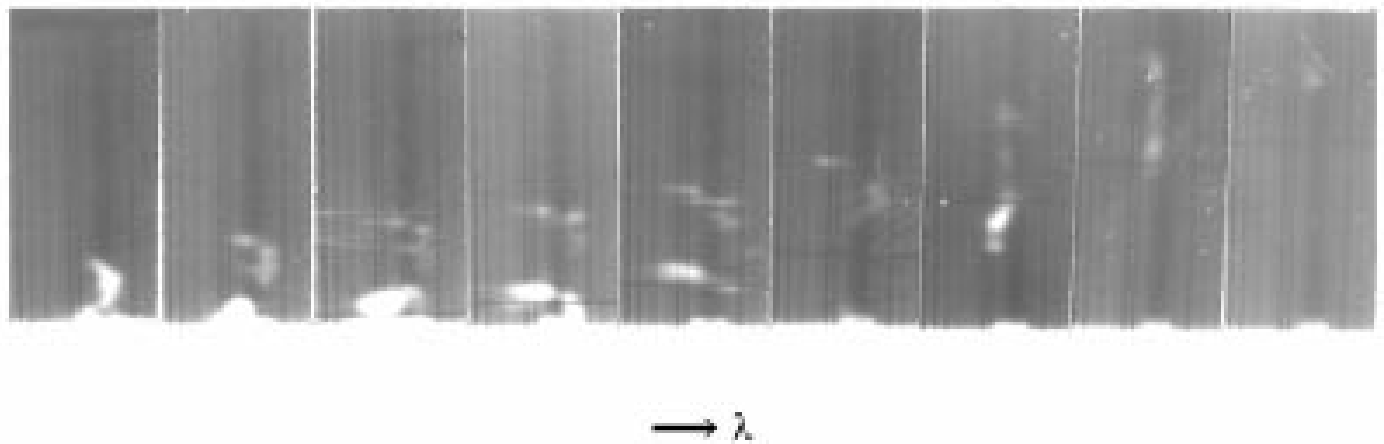

Fig. 1. The $\mathrm{H}_{\alpha}$ filtergrams $(0919-0947)$ of the eruptive prominence on 29 August 1990 and its partial $\mathrm{H}_{\gamma}$ spectral pictures (0927 - 0931UT). The black and thick lines in the filtergrams of 092744, 092858, and 093011UT are the images of the slit. The time order of the $\mathrm{H}_{\gamma}$ spectral pictures is from the left th the right in the pictures

and Leka (1993). The emerging process occurred before the maximum of the flare. At 0923UT, the left loop leg became thicker, and the materials in it continuously ascended along it. After reaching the zenith, they dropped downwards in succession not along the right loop leg, so the second loop structure was formed. Comparing the pictures at $0923 \mathrm{UT}$ and at $0927 \mathrm{UT}$, we estimate the falling velocity at $0923 \mathrm{UT}$ at about $90 \mathrm{~km} / \mathrm{s}$, the velocity of the materials hitting the solar surface at $0927 \mathrm{UT}$ at more than $163 \mathrm{~km} / \mathrm{s}$. The picture at $092744 \mathrm{UT}$ shows that the shape of the prominence is rather complicated, but one can still discern the emerging loop structure, the second loop structure, and what seems to be a third loop structure forming. At $092858 \mathrm{UT}$, the prominence reached climax, the 
third loop structure was formed, and the emerging loop was snapped at the top. The rupture may be due to the decaying of the prominence, and not the result of "progressive reconnection of elementary flux tubes of two (or more) interacting loops" as suggested by Simberova et al. (1993) and Yurchisin (1994). After that, the altitude of the prominence decreased, and the prominence gradually decayed. At 0940UT, the prominence became a regular arch structure, which made nearly a right angle with the solar surface.

In the decay stage, the obvious variation is the interruption at the vault of the arch, the materials descending along the two legs of the arch, but the outline of the arch not obviously changed. We measured the mean falling velocity at about $83 \mathrm{~km} / \mathrm{s}$ for the left leg and $74 \mathrm{~km} / \mathrm{s}$ for the right. The decay of the left leg is different from that of the right, indicating that the physical condition inside the left leg is different from that inside the right. Perhaps the material of the right is denser than the left, so that the gas pressure of the right is larger than at the left, reducing the falling velocity of the right.

We measured the altitudes of the prominence at different times, and dealt with these data with regresssion analysis. Altitudes $h$ (in kilometers) and the time $t$ (in minutes) have the linear relation:

$h=38439.9+4259.7 t$

$h=102379-2024.5 t$.

Equation (1) corresponds to the rising stage of the prominence, i.e. the formation of the prominence; Eq. (2) reflects the descending stage, the decaying of the prominence. Figure 2 gives the comparison of the measured values and the theoretical regression lines.

Figure 2 shows that the altitude of the prominence uniformly increased with an average velocity of about $71 \mathrm{~km} / \mathrm{s}$, then evenly decreased with an average velocity of about $33.7 \mathrm{~km} / \mathrm{s}$. The formation time is about half as much as the decay time.

\subsection{Source of the material}

The problem of filament formation has always been vexations, and the situation has been exacerbated by the caution of observers and the predilections of theoreticians. A prominence is formed by the emergence of a bright knot at the solar limb, but observers have been loath to say that prominences, hence filaments, emerge from the chromosphere. In most limb observations, prominence formation could be interpreted as resulting either by the condensation of matter from the corona or by the emergence of a heavily-laden flux rope from the chromosphere (Rust \& Kumar 1994).

The formation stage of the prominence indicates that the mass in the prominence was supplied during the emergence of helical flux ropes, i.e. it was lifted bodily out of the chromosphere. We do not believe that filaments or prominences condense from the corona or are formed by siphoning mass from the chromosphere, as already argued by Rust \& Kumar (1994).

\subsection{The explanation of observations}

The net effect of flux rope emergence and spiral accumulation in filaments or prominences is to eject spiral from the Sun. This process is a necessary mechanism that releases spiral generated inside the Sun (Rust \& Kumar 1994). The occurrence of the flare triggered the spiral ejection. With the development of the flare, the material ejection became more and more violent. Because the material ejection was intermittent, the ejected materials formed different trace, and the tracks became ever larger. This is why the three loop structures were formed during the flare, and the prominence reached the maximum height about 10 minutes later than the flare reached the maximum. We estimate the mean velocity of the ejected materials to be about $82 \mathrm{~km} / \mathrm{s}$ (the velocity $71 \mathrm{~km} / \mathrm{s}$ is its vertical component). The falling velocity is very large (about $90 \mathrm{~km} / \mathrm{s}$ at 0923UT). The value is impossibly in good agreement with that expected from the simplest model: a free-fall motion along a stationary loop. We suggest that the following process exist:

The shape of a prominence reflects the trend of the magnetic field lines or the structure of the magnetic field (Zhang 1992). According to the morphological features of the prominence, we propose a likely model to illustrate the observations of the prominence (see Fig. 3). First, a magnetic flux tube emerged to form a loop structure (see Fig. 3a and the $\mathrm{H}_{\alpha}$ filtergram of 0919UT in Fig. 1). The newly emerging magnetic tube squeezed the magnetic field lines of the ambient corona. The observations suggest that we witnessed a reconnection process between the constantly-emerging magnetic field lines and the squeezed magnetic field lines of the adjacent corona. The possible scenario is shown in Fig. 3b. There are two reasons to make such a suggestion. One is that the falling velocity is very large (about $90 \mathrm{~km} / \mathrm{s}$ at $0923 \mathrm{UT}$ ). There must be another acceleration in addition to gravity. We suggest that the falling velocity is largely due to gravity, but mainly due to the acceleration caused by the reconnection of the magnetic lines. The other reason is that the filtergrams at $092744 \mathrm{UT}$ and $092858 \mathrm{UT}$ show a spike $\mathrm{M}$ at the top of the prominence (see Fig. 1). Maybe the spike $M$ is the product of the reconnection. The reason why the spike did not escape from the prominence to produce a CME event is that the motion of the spike was hindered by the magnetic lines of the ambient corona. This physical scenario is analogous to that given by Wang et al. (1989).

Schmieder et al. (1995) analysed $\mathrm{H}_{\alpha}$ and SXT data of a recurrent surge and suggested that surges are produced by magnetic reconnection between a twisted cool loop and 


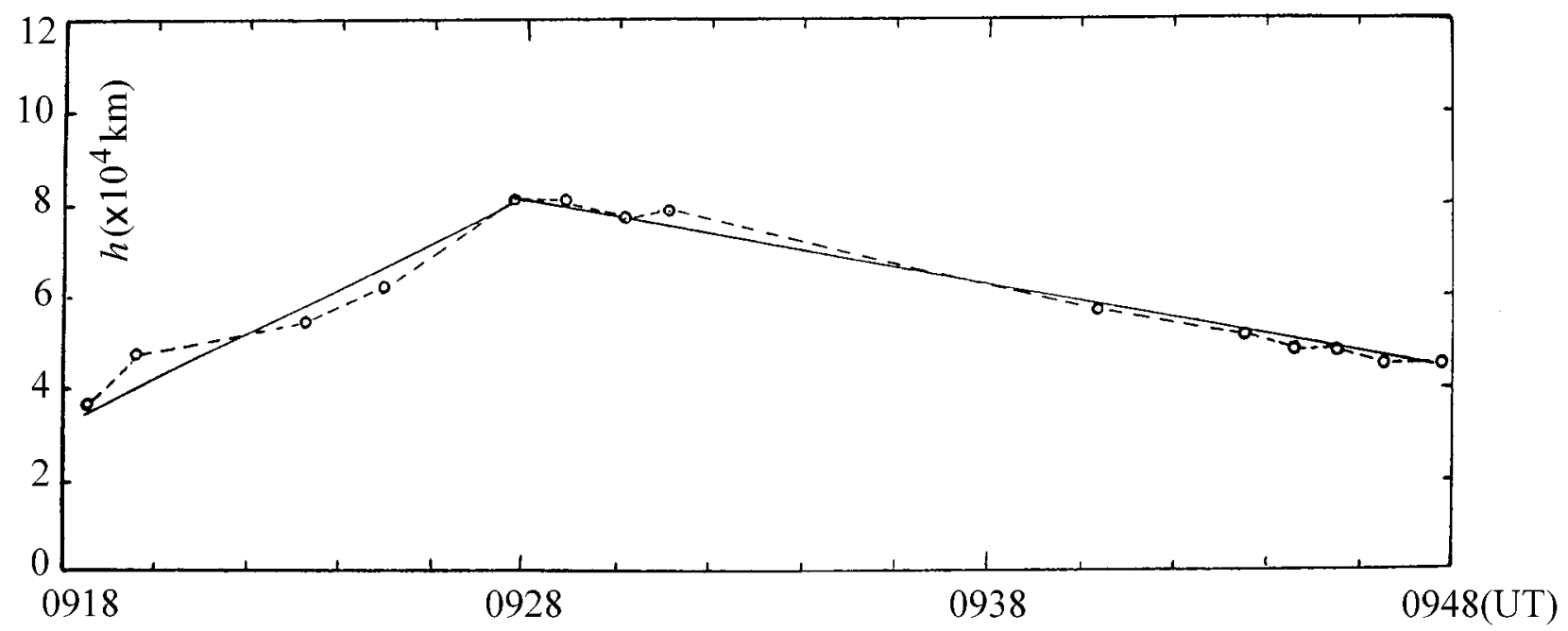

Fig. 2. Variation of the prominence altitude with time. The solid line and the dashed line correspond to the regression line and the observational curve respectively

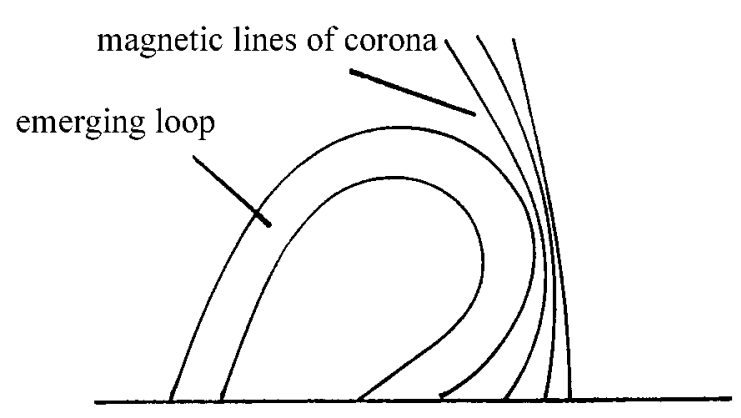

a

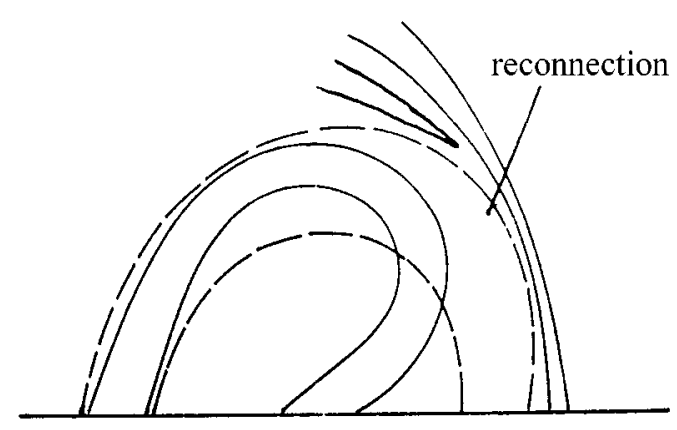

b

Fig. 3. The suggested magnetic configuration for the prominence and the ambient corona

open field lines. Our explanation of the prominence is very similar to their results (Schmieder et al. 1995).

\section{Spectral diagnoses}

The prominence was scanned with $\mathrm{H}_{\gamma}$ line from the left to the right of the filtergrams of Fig. 1, and the slit was parallel to the left edge of each filtergram. The scanning time was 0927UT to 0930UT. Combining the $\mathrm{H}_{\gamma}$ spectra and $\mathrm{H}_{\alpha}$ filtergrams, we can infer that the materials in the left leg of the eruptive prominence spiraled to rise. This is consistent with the results obtained in section two. The $\mathrm{H}_{\gamma}$ spectra obtained were measured with the PDS microdensitometer at the Purple Mountain Observatory. Comparing the spectra with the $\mathrm{H}_{\alpha}$ filtergrams, we can locate the position of each $\mathrm{H}_{\gamma}$ line in the filtergrams of the prominence. After calculating the center-of-gravity position $(\lambda)$ of each chosen line profile and then the Doppler shift $\Delta \lambda_{0}\left(\Delta \lambda_{0}=\lambda-\lambda_{0}, \lambda_{0}=4340 \AA\right)$, we obtained the velocity along the line of sight $V_{\|}\left(V_{\|}=C \Delta \lambda_{0} / \lambda_{0}\right)$.
Figure 4 gives the distribution of the line-of-sight velocity, which indicates that the velocity increased from the bottom of the left leg to the top. After the materials in the left leg reached the climax, those dropped out in succession and did not spiral. Figure $4 \mathrm{~b}$ summarises the configuration of the velocity distribution. The materials are usually frozen in the magnetic field of a prominence, and they move mostly along the magnetic lines. So we think of the magnetic field of the prominence helical, stretched primarily along the axis, and tied to the photosphere only at the end of the prominence. There is growing evidence that filaments (or prominences) emerge from the photosphere already twisted (Rust \& Kumar 1994; Yang et al. 1988; Liggett \& Zirin 1984; Vrsnak et al. 1991). The prominence motion shown in Fig. 4b may be called "right-hand screw" when it is viewed by a hypothetical observer in the chromosphere. Rust \& Kumar (1994) infer that the magnetic fields of prominences are twisted in a systematic way: right-hand helices predominate in the south and left-hand helices predominate in the north. The result 
obtained by spectral analyses confirms their inference. The velocity increased perhaps because (1) the angular velocity increased from the bottom to the top due to the release of the torsion of the prominence; (2) the angular velocity may be constant, but the radius of the leg increased from the bottom to the top.
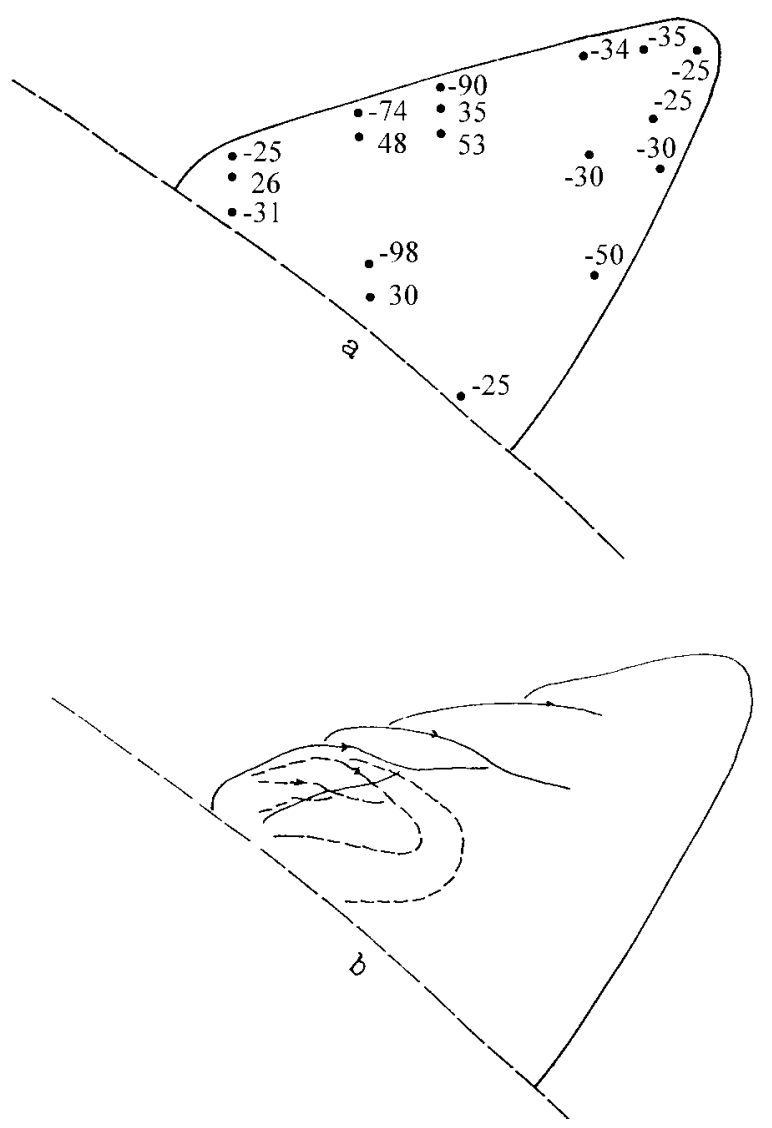

Fig. 4. a) Distribution of the velocity (in $\mathrm{km} / \mathrm{s}$ ) along the line of sight. b) Sketch for explanation of the velocity distribution. The arrow shows the direction of the material motion

\section{Conclusions}

In this paper, a study of the eruptive prominence on 29 August 1990 has been presented. The $\mathrm{H}_{\alpha}$ filtergrams and the $\mathrm{H}_{\gamma}$ spectra of the prominence are given to study its morphological evolution and kinematic characteristics. The main results are (1) the rising velocity of the altitude of the prominence (about $71 \mathrm{~km} / \mathrm{s}$ ) is over twice as large as the falling velocity (about $33.7 \mathrm{~km} / \mathrm{s}$ ), the formation time of the prominence is about half as much as the decay time.
The formation stage of the prominence demonstrated that the materials in the prominence were supplied during the emergence of helical flux tubes; (2) the materials in the prominence spired to rise, then after reaching the climax, they dropped in succession, but did not spiral. The velocity along the line of sight increased from the bottom to the top of the prominence. We suggest but do not confirm that the angular velocity increased from the bottom to the top due to the release of torsion of the prominence. During the period of the development of the prominence, three loop structures were formed, and we think that the material ejection was intermittent.

Acknowledgements. We thank the referee for very useful comments. This paper has been prepared on the VAX-8350 Computer at Yunnan Observatory, and we both thank the clerks of the VAX-8350 Computer Group. This work is supported by the President's Foundation of the Chinese Academy of Sciences.

\section{References}

Hirayama T., 1979, IAU Colloq. 44, 4

Hirayama T., 1985, Solar Phys. 100, 415

Leka K.D., 1993, presented at the 24th Meeting of the A\&AS Solar Phys. Div., Palo Alto, Calif.

Liggest M., Zirin H., 1984, Solar Phys. 91, 259

NOAA, 1990a, Solar-Geophysical Data, Comprehensive Reports, Part II, 558, 32

NOAA, 1990b, Solar-Geophysical Data, Comprehensive Reports, Part II, 558, 102

Poland A.I., 1986, Coronal and Prominence Plasmas, NASA Conf. Publ. 2442, Nat. Aerospace Admin., Washing, DC

Priest E., 1989, Dynamics and Structure of Quiescent Solar Prominences. Kluwer Academic Publishers, Dordrecht, Holland

Rust D.M., Kumar A., 1994, Solar Phys. 155, 69

Ruzdjak V., Tandberg-Hassen E., 1990, Dynamics of Quiescent Prominences, Lect. Notes Phys. 363. Springer-Verlag, New York

Schmieder B., Shibata K., van Driel-Gesztelyi L., Freeland S., 1995, Solar Phys. 156, 246

Simberova S., Karlicky M., Svestka Z., 1993, Solar Phys. 146, 343

Tanaka K., 1991, Solar Phys. 136, 133

Vrsnak B., Ruzdjak V., Rompolt B., 1991, Solar Phys. 136, 151

Wang J.X., et al., 1989, Chin. J. Astrophys. 9, 260

Xuan J.Y., Lin J., 1993, Solar Phys. 144, 307

Yang H.S., Hong Q.F., Ding Y.J., 1988, Solar Phys. 117, 57

Yurchisin V.B., 1994, Solar Phys. 154, 199

Zhang Z.D., 1992, Solar Phys. Science Press, Beijing, China, p. 341 